\title{
Small Intestinal Multifocal Stenosing Ulceration
}

\author{
Hugh James Freeman ${ }^{1}$
}

Received: 13 April 2015/Accepted: 16 April 2015/Published online: 5 May 2015

(C) Springer Science+Business Media New York 2015

\section{Background and Significance}

A broad range of ulcerative intestinal inflammatory disorders may often initially manifest as abdominal pain. In this issue of Digestive Diseases and Sciences [1], Chung and colleagues from South Korea describe a multicenter case series of "cryptogenic multifocal ulcerous stenosing enteritis" or CMUSE, defined using modern endoscopic methods for small intestinal mucosal evaluation and followed up for an extended period. Through these observations, the authors provide novel information regarding the long-term natural history of CMUSE, a specific syndrome initially described more than a half-century ago largely, although not entirely, in the French literature [2-6]. In these early reports, CMUSE was typically associated with multiple shallow mucosal ulcerations, usually in the jejunum or proximal ileum. Often, steroid responsiveness was noted, although surgical treatment was sometimes eventually needed. In the present series, the investigators were able to evaluate a group of patients not treated with biological agents. Differentiation from other entities, specifically Crohn's disease, was largely based on location of the ulceration and proximal small intestinal strictures, different from most, but not all patients with Crohn's disease with disease usually localized in distal small intestine and colon [7]. An absence of the typical granulomatous inflammatory change reported in some, but not all resected intestinal materials from Crohn's disease patients [7], was

Hugh James Freeman

hugfree@shaw.ca

1 Department of Medicine (Gastroenterology), University of British Columbia Hospital, 2211 Wesbrook Mall, Vancouver, BC V6T 1W5, Canada also noted. Now, as in the present study, the use of newer imaging modalities was documented in CMUSE, including double-balloon enteroscopy. This technique offers the opportunity to extend standard endoscopic methods for visualization to further explore the small intestine, and possibly add to more precise differentiation of CMUSE from other causes.

\section{Addressing the Issues}

These investigators acknowledge that exclusion of other causes of small bowel ulceration was considered in their initial evaluation and on independent review of the entire clinical series by two of the authors. To properly predict the long-term natural history of any disorder, precise initial definition is crucial, since further understanding of this descriptive entity is critically dependent on accurate diagnosis.

Several entities, shown in Table 1, have been associated with small bowel ulceration and subsequent stricture formation [8]. In ulcerative (non-granulomatous) jejunoileitis, moderate-to-severe architectural changes occur with flattening of the villi, often associated with celiac disease, or as a complication of celiac disease per se. In patients with celiac disease, however, mucosal biopsy changes in untreated disease are characteristic (not diagnostic) along with concomitant tissue transglutaminase antibodies. Here, responsiveness to a gluten-free diet is essential. In some with celiac disease, a difficult-to-diagnose focal mucosal lymphoma might also be responsible for mucosal ulceration, with malignant lymphoma cells recognized at the edge of mucosal ulcers. In South Korea, celiac disease appears to be rare with only a single case recorded [9]. 
Table 1 Reported causes of multifocal small bowel ulceration and stenosis

\author{
Cryptogenic multifocal ulcerous stenosing enteritis (CMUSE) \\ Crohn's disease (with proximal small bowel involvement) \\ Complicated celiac disease (ulcerative non-granulomatous jejunoileitis, ulcerated mucosal lymphoma) \\ Infectious agents (eg., Campylobacter, Yersinia) \\ Medication induced (eg., pharmaceutical agents, NSAIDs; biological agents, ipilimumab) \\ Gastrin-secreting tumor with Zollinger-Ellison syndrome \\ Traumatic injuries (including endoscopic treatment and seat belt injury) \\ Ischemia (eg., collagen vascular diseases, vasculitis)
}

Adapted from [8]
Table 2 Features of CMUSE to differentiate from Crohn's disease

\author{
Lack of clinical and laboratory features of inflammatory syndrome \\ Absence of imaging or pathological features of a transmural inflammatory process \\ Absence of giant cell granulomatous inflammatory process in resected tissue \\ "Failure of fistulization" in spite of recurrent inflammatory episodes \\ Absence of disease elsewhere in the gastrointestinal tract (eg., stomach, colon) \\ Absence of perianal disease \\ Absence of most extraintestinal features typical of Crohn's disease (e.g., skin manifestations)
}

Adapted from [8]
A host of infectious agents (e.g., Campylobacter, Shigella, or Yersinia species) may cause small bowel ulcers, but most likely resolve completely without stricture formation. Still, because follow-up may be limited, information on long-term ulcerations of the small intestine after an infection is limited. Other infectious agents, including cytomegalovirus, Mycobacterium species, and Tropheryma whipplei, may also cause small intestinal ulcers, possibly through a different mechanism. Most infectious agents are amenable to precise diagnosis by evaluation of clinical materials, including tissue.

The list of medications that may cause small bowel ulceration continues to expand. Perhaps, most common are nonsteroidal anti-inflammatory drugs (NSAIDs) that may cause frank ulcers, mucosal erosions, strictures, and socalled diaphragm disease. Other classical agents include potassium tablets, gold, and chemotherapeutic agents. In recent years, ulceration in the small and large bowel may also result from monoclonal biological agents (e.g., ipilimumab). Peptic ulceration may also occur in unusual small intestinal sites due to a gastrin-secreting tumor or associated with heterotopic gastric mucosa.

Traumatic penetrating injuries or ingested foreign bodies (e.g., toothpicks) may ulcerate the small bowel. Sometimes, ulceration and stricture formation may occur following blunt abdominal trauma, such as seat belt injury following a motor vehicle accident. Increasingly, traumatic injuries may also result from endoscopic biopsies for diagnostic purposes and therapeutic interventions (e.g., cautery for polypectomy). Ischemic disease may also manifest with small bowel mucosal ulceration. Some of these may be associated with systemic disease including thrombotic diseases, Dego's disease, pseudoxanthoma elasticum, myeloproliferative disorders, antithrombin III deficiency, and inflammatory vasculitis associated with an occult collagen vascular disease (e.g., systemic lupus erythematosus or polyarteritis nodosa).

Perhaps, most intriguing and most important is differentiation of CMUSE from Crohn's disease, especially if the latter only appears to involve the small intestine. Crohn's disease, for example, may uncommonly occur in the proximal small bowel including either duodenum or jejunum without evidence of disease elsewhere [10], usually associated with other clinical and pathological features, as detailed in Table 2 [8]. At the same time, since precise diagnosis of Crohn's disease may be difficult, consideration of each of these entities, including CMUSE, is important to anticipating a clinical response to specific forms of therapy.

\section{Future Directions}

CMUSE is a syndrome that appears to have a distinct clinical and pathological profile; however, other overlapping features with some small intestinal disorders, particularly Crohn's disease, make differentiation difficult. Since both entities do not have either a precise or definitive diagnostic signature, treatment schemes based on modern clinical trials may be misleading. Development of more 
precise diagnostic tools for either entity appears to be evolving, especially with new or novel imaging methods critical to future treatment. Further appreciation for their long-term natural history in larger cohorts will likely help symptomatic management, but definition of their precise causes and pathogenesis should remain the focus of fundamental investigation.

\section{References}

1. Chung SH, Park SU, Cheon JH, et al. Clinical characteristics and treatment outcomes of cryptogenic multifocal ulcerous stenosing enteritis in Korea. Dig Dis Sci. (Epub ahead of print). doi:10. 1007/s10620-015-3595-y.

2. Rocha A, Artigas V. Stenosing ulcerous disease of the jejunoileum. Arch Mal Appar Dig Mal Nutr. 1959;48:1230-1236.

3. Debray C, Besancon F, Hardouin JP, Martin E, Marche C, Khoury K. Cryptogenic plurifocal ulcerative stenosing enteritis. Arch Mal Appar Dig Mal Nutr. 1964;53:193-206.
4. Chagnon JP, Devars du Mayne JF, Marche C, Vissuzaine C, Cerf M. Multifocal cryptogenetic stenosing enteritis: an autonomous entity? Gastroenterol Clin Biol. 1984;8:808-813.

5. Perlemuter G, Chaussade S, Soubrane O, et al. Multifocal stenosing ulcerations of the small intestine revealing vasculitis associated with C2 deficiency. Gastroenterology. 1996;110:16281632.

6. Matsumoto T, Iida M, Matsui T, et al. Non-specific multiple ulcers of the small intestine unrelated to non-steroidal anti-inflammatory drugs. J Clin Pathol. 2004;57:1145-1150.

7. Freeman HJ. Application of the Montreal classification for Crohn's disease to a single clinician database of 1015 patients. Can J Gastroenterol. 2007;21:363-366.

8. Freeman HJ. Multifocal stenosing ulceration of the small intestine. World J Gastroenterol. 2009;15:4883-4885.

9. Gweon TG, Lim CH, Byeon SW, et al. A case of celiac disease. Korean J Gastroenterol. 2013;61:338-342.

10. Schuffler MD, Chafee RG. Small intestinal biopsy in a patient with Crohn's disease of the duodenum. The spectrum of abnormal findings in the absence of granulomas. Gastroenterology. 1979;76:1009-1014. 